\title{
Reinhard Marx
}

\section{Transnationale karitative Hilfe unter Terrorismusverdacht*}

\section{Problemaufriss}

Die Zunahme der nichtstaatlichen transnationalen Vernetzung hat dazu geführt, dass die Gesamtmenge weltumspannender Beziehungen inzwischen größer ist als die Summe nationalstaatlicher Gesellschaften, ${ }^{1}$ sodass es im verstärkten Maße der Herausbildung transnationaler Öffentlichkeiten bedarf, in denen im nationalen Rahmen gesellschaftlich herausgebildete Wertvorstellungen und Praxen überprüft und legitimiert werden können. ${ }^{2}$ Diese insbesondere die universelle Verständigung über rechtliche Normen und Verfahren prägende Tendenz hat auch Auswirkungen auf transnationale humanitäre Hilfe. Staatliche Entwicklungshilfe ist heute auf die Hilfe nichtstaatlicher Organisationen (NGOs) angewiesen. Ohne deren Hilfe könnte die Nothilfe in vielen Ländern der Welt kaum mehr abgewickelt werden, zudem NGOs in bewaffneten innerstaatlichen Konflikten flexibel und pragmatisch mit den bestimmenden politischen Kräften kooperieren können. ${ }^{3}$ Humantäre Hilfe von außen bedingt notwendigerweise die Kooperation mit allen wichtigen Akteuren innerhalb eines Konfliktgebietes, damit die Hilfe an bedürftige Menschen vor Ort gelangt. Zur Sicherstellung wirksamer transnationaler Hilfe müssen alle militärischen wie nichtmilitärischen Akteure einbezogen werden. Andererseits kann angesichts der entgrenzten Gewalt in derartigen Konflikten keine präzise Grenzlinie zwischen militärischen und terroristischen Kräften gezogen werden. Durch westliche Geberländer und Regierungen vorgenommene Zuschreibungen sind ohnehin problematisch, wenn sie mit universellem Durchsetzungsanspruch auftreten. Was dem Westen als „terroristisch“ erscheint, muss nicht in der betroffenen Region und insbesondere von der Bevölkerung in den Zielländern humanitärer Hilfe notwendigerweise so gesehen werden.

Wird transnationale karitative Hilfe im Lichte dieses globalen, dynamischen Kontextes gesehen, kann die 2004 vom Bundesverwaltungsgericht entwickelte Rechtsprechung im Vereinsverbotsrecht, sollten ihr andere westliche Länder folgen, deren Wirksamkeit langfristig beschädigen. Am Beispiel der vom Bundesinnenministerium im Juli 2002 verbotenen Al Aqsa, die an Sozialvereine der HAMAS Hilfe geleistet hatte, erweiterte das Bundesverwaltungsgericht seine Rechtsprechung zum Begriff der Unterstützung von Vereinigungen, die sich gegen den Gedanken der Völkerverständigung (Art. 9 Abs. 2 GG) richten. Es dehnte nicht nur diesen materiellen Verbotsgrund aus, sondern erweiterte den ohnehin schon konturenlosen Begriff der Unterstützung ${ }^{4}$ durch Einführung eines Unterstützungsbegriffs, der die mittelbaren Akzeptanz- und Entlastungsvorteile für terroristische Organisationen einschließt, die mit zweckgebundener transnationaler

\footnotetext{
Der Autor hat die Internationale Humanitäre Hilfsorganisation (IHH) im Verfahren vertreten.

1 Albrow, Abschied vom Nationalstaat, 1996, S. 257.

2 Honneth, Das Recht der Freiheit, 2011, S. $564 \mathrm{ff}$.

3 Ludermann, Privater Arm der Geberstaaten?, in: Brühl u.a. (Hrsg.), Die Privatisierung der Weltpolitik, 2001, S. 174 (187).

4 S. hierzu Marx, Aufenthalts-, Asyl- und Fluchtlingsrecht, 4. Aufl., 2011, S. 815 ff.; Marx, ZAR 2002, 127.
} 
karitativer Hilfe an soziale Einrichtungen möglicherweise verbunden sein können. ${ }^{5}$

Es ist evident, dass mit einem derart begrifflich unscharfen Unterstützungsbegriff transnationale karitative Hilfe mühelos als „terroristische Unterstützung“ denunziert und zur Grundlage eines Vereinsverbots gemacht werden kann. Selbstverständlich stehen anerkannte Organisationen, wie etwa die Caritas, das Diakonische Werk, die Welthungerhilfe, das Rote Kreuz oder Oxfam, und erst recht staatliche Entwicklungshilfe von vornherein außerhalb jeglichen Verdachts, auch wenn ihre transnationale Hilfe wegen nicht zu vermeidender Kontakte mit Gruppierungen, die als „terroristisch“ eingestuft werden, nach den Kriterien der Rechtsprechung als Unterstützung dieser Vereinigungen gewertet werden kann. Ins Visier der Verbotsbehörde geraten vielmehr karitative Vereinigungen, denen in unserer Gesellschaft wegen ihrer Einbindung in muslimische Migrantenstrukturen und ihres Fokus auf islamische Zielländer die erforderliche gesellschaftliche Anerkennung fehlt. Die Befürchtung, dass die Wirksamkeit transnationaler Hilfe durch eine derartige Rechtsprechung langfristig beschädigt werden kann, wird dadurch verstärkt, dass seit dem 11. September 2001 die erforderliche Gefahrenabwehr zunehmend auch durch islamophob motivierte gesellschaftliche und staatliche Zuschreibungen und Feindbilder beeinflusst wird, andererseits aber gerade islamische Länder wegen ihres besonderen Bedarfs an transnationaler humanitärer Hilfe im Fokus dieser Hilfe stehen. ${ }^{6}$ Häufig sind es insbesondere islamische NGOs wie etwa die türkische Internationale Humanitäre Hilfsorganisation ( $\mathrm{IHH})$, die in zerstörten und extrem gefährlichen islamischen Zielländern wie Somalia humanitäre Hilfe leisten. ${ }^{7}$ Diese Hilfe ist in vielen Ländern der islamischen Welt unverzichtbar und umfasst 57 Zielländer, in denen die Bevölkerung durch Naturkatastrophen und bewaffnete Konflikte bedroht ist und dringend humanitärer Hilfe bedarf. ${ }^{8}$

Im unmittelbaren zeitlichen Zusammenhang mit der durch die türkische IHH im Mai 2010 durchgeführten Durchbrechung der israelischen Blockade von Gaza wurde die deutsche IHH, ${ }^{9}$ die in Verbindung mit der von den Verfassungsschutzbehörden beobachteten Islamischen Gemeinschaft Milli Görüs (IGMG) steht, durch das Bundesinnenministerium am 23. Juni 2010 verboten. Wohl ermutigt durch die Al Aqsa-Rechtsprechung wurde die Prozessführung der Verbotsbehörde weniger durch belastbare Tatsachen als vielmehr weitgehend durch assoziativ begründete islamophobe Feindbilder geprägt. Es drängt sich dadurch der Eindruck auf, dass die Verbotsbehörde Instrumente polizeirechtlicher Gefahrenabwehr zur Einschüchterung islamischer Gemeinschaften im Bundesgebiet zum Einsatz bringen will. Die deutschen Wohlfahrtsverbände bleiben in Deckung, obwohl es um ein Kernanliegen ihres Mandates geht. Feindzuschreibungen der Verfassungsschutzbehörden zeitigen ihre Wirkung. Mit „Schmuddelkindern" möchte man nicht in Verbindung gebracht werden.

Formale Begründung für das Verbot der IHH ist, dass diese im Rahmen ihres weltweiten Programms auch projektgebundene Spenden für rund 3000 Waisenkinder in Gaza und entsprechende Patenschaften insbesondere türkischer Migranten in Deutschland über Sozialvereine der HAMAS abgewickelt hatte. Da-

6 S. hierzu Konferenzbericht „Development Cooperation in the Context of Islam: Working together for constructive Politics and Partnerships“, Islamic Relief Deutschland (IRD) \& Welthungerhilfe, Januar 2010.

7 Selbert, Katastrophe in Somalia. Islamische Welt will helfen, www.tagesspiegel.de, 29. Juli 2011.

8 Konferenzbericht „Development Cooperation in the Context of Islam (Fn. 6), S. 7.

9 Die deutsche IHH ist keine Unterorganisation der türkischen IHH. Vielmehr ist die türkische Partnerorganisation der deutschen IHH die Vereinigung Cansuyu. 
durch habe sie terroristische Angriffe der HAMAS gegen Israel unterstützt. Die Palästinahilfe der IHH hat jedoch nur eine geringe Priorität innerhalb ihrer transnationalen karitativen Hilfe in muslimische Zielländer. Zudem hat die IHH stets strikt darauf geachtet, dass ihre Hilfslieferungen projektbezogen durchgeführt wurden, hat Patenschaften hier lebender Arbeitsmigranten für palästinensische Waisenkinder in Gaza und den Westbanks vermittelt und war mangels eigener organisatorischer Strukturen in Palästina auf dort tätige Sozialvereine für die Vermittlung dieser Projekte angewiesen. Insbesondere nach der Operation „Gegossenes Blei“ Ende 2008/Anfang 2009 hatten viele türkische Migranten die IHH aufgefordert, mehr praktische Hilfe für Waisenkinder in Gaza zu leisten. Folge des Verbots der IHH ist, dass 3000 palästinensische Waisenkinder nicht weiter gefördert werden können. Inzwischen hat das Bundesverwaltungsgericht nach zwei mündlichen Verhandlungen am 18. April 2012 das Verbot bestätigt. ${ }^{10}$ Die besorgniserregende Anwendung polizeirechtlicher Instrumente zur Feindabwehr in einem sensiblen gesellschaftlichen Kontext macht zuallererst eine eindeutige gesellschaftliche Reaktion der Mehrheitsgesellschaft erforderlich. Darüber hinaus bedarf es einer verfassungsrechtlichen wie auch verwaltungs- und insbesondere polizeirechtlich begründeten Kritik dieser Verbotspraxis und Rechtsprechung.

\section{II. Überschießender Präventionsbegriff}

Ausgangspunkt der Rechtsprechung ist Art. 9 Abs. 2 GG. Danach sind Vereinigungen verboten, deren Zweck oder deren Tätigkeit sich gegen den Gedanken der Völkerverständigung richten. Die 2004 geänderte Rechtsprechung des Bundesverwaltungsgerichts zum Begriff der Zuwiderhandlung gegen den Gedanken der Völkerverständigung beruht auf einem Unterstützungsbegriff, der nicht mehr am Ziel der Gefahrenabwehr orientiert ist, sondern weit über diese hinausgeht. Dadurch wird die ohnehin bestehende Tendenz in der Rechtsprechung verstärkt, seinen objektiven Tatbestand ins Uferlose auszudehnen. In der Rechtsprechung des 1 . und 5. Senats des Bundesverwaltungsgerichts wird ausdrücklich eingeräumt, dass die Auslegung und Anwendung des Unterstützungsbegriffs nach Maßgabe eines tatbestandlich weiten Unterstützungsbegriffs erfolgt. ${ }^{11}$ Die Al Aqsa-Rechtsprechung des 6. Senats bemüht sich erst gar nicht mehr um eine dogmatische Begriffsklärung nach Maßgabe des Gefahrenabwehrrechts, sondern entwickelt losgelöst von dessen rechtsstaatlichen Schranken einen weit ausgreifenden Ansatz:

Der Wortlaut von $\$ 3$ Abs. 1 Satz 1 VereinsG, der Art. 9 Abs. 2 GG umsetzt, gibt keine Auskunft darüber, welche Art der Gefahr für die dort bezeichneten Rechtsgüter vorliegen muss, um ein Vereinsverbot zu rechtfertigen. Er fordert lediglich, dass Tätigkeit und Zweck der Vereinigung, die einen Verein unterstützt, dessen Zweck und Tätigkeit sich gegen den Gedanken der Völkerverständigung richtet, sich „gegen“ diesen Gedanken „richten.“ Es ging dem Verfassungsgeber darum, den Grundrechtsschutz für Organisationen auszuschließen, deren Tätigkeit elementaren Grundsätzen der Völkerverständigung zuwiderlaufen. Er war dabei von der Erkenntnis geleitet, dass derartige Aktivitäten besonders gefährliche 
Entwicklungen auszulösen vermögen, wenn sie sich auf eine gemeinschaftliche Basis stützen können. "12 Daher setzt das Erfordernis, dass sich die Vereinigung gegen den Gedanken der Völkerverständigung richten muss, die Feststellung von Tatsachen voraus, die auf eine Tätigkeit der Vereinigung mit dem Ziel unmittelbarer Verwirklichung hierauf gerichteter Absichten hinweisen. ${ }^{13}$ Wesentlich für die Tatsachenfeststellung in diesem Zusammenhang ist, dass sich die Tätigkeit der Vereinigung kämpferisch-aggressiv gegen diese Ziele wendet. ${ }^{14}$ In der Rechtsprechung des 6. Senates des Bundesverwaltungsgerichts wurde dieses Erfordernis aufgegeben und darüber hinaus im Rahmen transnationaler karitativer Hilfe ein Unterstützungsbegriff eingeführt, der auch bloße mittelbare bis denkbare Auswirkungen derartiger Hilfe diesem Begriff zuordnet, also auf das Erfordernis zweckgerichteter und unmittelbarer gefährdender Handlungen verzichtet, demgegenüber auch zweckgebundene karitative Hilfslieferungen in diesen Begriff ausdrücklich einschließt. ${ }^{15}$

Nach dem Grundsatz der Verhältnismäßigkeit ist ein Grundrechtseingriff nur dann verhältnismäßig, wenn eine unmittelbare, aus erkennbaren Umständen herleitbare Gefährdung des geschützten Rechtsguts gegeben ist. Die erforderliche Eingriffsschwelle muss zwar von Verfassungs wegen nicht notwendig eine gegenwärtige Gefahr im überkommenen Sinne sein, darf aber die einer konkreten Gefahr nicht unterschreiten. ${ }^{16}$ Wird lediglich eine abstrakte Gefahr gefordert, droht eine zu weitgehende Einschränkung der grundrechtlich verbürgten Assoziationsfreiheit, wie gerade die Bewertung transnationaler Karitas durch das Bundesverwaltungsgericht verdeutlicht: Eine abstrakte Gefahr ist gegeben, wenn eine generell-abstrakte Betrachtung für bestimmte Arten von Verhaltensweisen oder Zuständen zu dem Ergebnis führt, dass mit hinreichender Wahrscheinlichkeit ein Schaden im Einzelfall einzutreten „pflegt“ und daher Anlass besteht, die Gefahr mit generell-abstrakten Mitteln, also einem Rechtssatz, zu bekämpfen. ${ }^{17}$ Wenn es nach Ansicht des Bundesverwaltungsgerichts ausreicht, dass karitative Zuwendungen an Sozialvereine generell geeignet seien, positive Auswirkungen auf den militärisch terroristischen Bereich der HAMAS zu entfalten, ${ }^{18}$ rechtfertigt danach bereits das Bestehen einer abstrakten Gefahr ein Vereinsverbot. Klarstellend hebt das Bundesverwaltungsgericht ausdrücklich hervor, es bedürfe keiner Feststellungen dazu, dass zweckentsprechend für soziale Zwecke gespendete Gelder in den militärischen Bereich von HAMAS geflossen seien. ${ }^{19}$ Allein eine abstrakte Gefahr vermag ein Vereinsverbot nicht zu rechtfertigen. Die in Art. 9 Abs. 1 GG verbürgte Assoziationsfreiheit gewährleistet dem Einzelnen nicht nur das Recht zum Zusammenschluss in Vereinen, sondern auch diesen Vereinigungen selbst das Recht auf Sicherung ibres Bestehen ${ }^{20}$ und schützt damit insbesondere vor einem Verbot des Vereins. ${ }^{21}$ Das Verbot ist der denkbar schwerste Eingriff in dieses Grundrecht. Daher wird seiner Bedeutung und der Schwere des Eingriffs nur angemessen Rechnung getragen, wenn eine konkrete 
Gefahr für das betroffene Schutzgut als Voraussetzung für den Eingriff festge-

stellt wird. Das Spannungsverhältnis zwischen Freiheit und Sicherheit darf nicht einseitig zugunsten eines präventiv ausgerichteten Sicherheitsbegriffs aufgelöst werden, der bereits abstrakte Gefährdungen einbezieht, die aus jeglicher Art von Kontaktaufnahme, Berührung und Kommunikation mit Organisationen entstehen können, die in Konfliktgebieten die humanitäre Versorgung der Bevölkerung sicherstellen und irgendwie mit Organisationen verbunden sind, die dort als Konfliktbeteiligte agieren. Bei religiös motivierter Karitas ist im grundrechtsverstärkenden Sinne der besonders hohe Rang der Assoziationsfreiheit in seiner religiösen Dimension zu berücksichtigen. ${ }^{22}$ Der überschießende Präventionsbegriff des Bundesverwaltungsgerichts verkennt den besonderen Charakter des Grundrechts und führt dazu, dass unabdingbar erforderliche transnationale karitative Hilfe als „terroristische Unterstützung“ kriminalisiert und die nichtprofitorientierten, aus muslimisch karitativer Motivation handelnden Helfer in der Öffentlichkeit zu „Komplizen von Terroristen“ stigmatisiert werden, wie das $I H H$-Urteil anschaulich verdeutlicht.

\section{Völkerrechtsfreundliche Karitas als Verletzung der Völkerfreundschaft}

Das Bundesverwaltungsgericht macht den Begriff der Völkerverständigung für eine wirksame Gefahrenabwehr nicht nur unbrauchbar, sondern dreht ihn darüber hinaus auch begrifflich um. Die Befreiung von den lästigen Schranken des Polizeirechts enthebt es zugleich von den normativen Bindungen der Verfassung und führt zur Stigmatisierung völkerrechtlich förderungswürdiger Karitas im Namen des Schutzes der Völkerverständigung. Wie das geht? Die hierfür notwendige juristische Transformation eines Begriffs durchläuft mehrere Stufen: Zunächst gibt das Bundesverwaltungsgericht dem Begriff der Völkerverständigung einen neuen Inhalt. Der Gedanke der Völkerverständigung reiche weiter als das friedliche Zusammenleben der Völker. Er wolle verhindern, dass „Gewalt in das Verhältnis von Völkern hineingetragen“ werde. ${ }^{23}$ War der Begriff bis 2004 in Anlehnung an das Angriffsverbot im Sinne von Art. 2 Nr. 4 UN-Charta, Art. 26 Abs. 1 Satz 1 GG am friedlichen Zusammenleben der Staaten ausgerichtet, wandert der Blick nunmehr von den Konflikten zwischen staatlich verfassten Völkern zu den verfeindeten Ethnien innerhalb der Staaten und ergreift Partei für die den Staat beherrschende Ethnie. Das Vereinsverbot will nunmehr den Frieden innerhalb von national verfassten Gesellschaften von außen sichern. Potenziell steht damit die gesamte Weltunordnung im Fokus des nationalen Polizeirechts. Eine legitime Herkulesaufgabe? Zumindest tangiert eine derartige Auslegung das grundlegende völkerrechtliche Prinzip der Nichteinmischung (Art. 2 Nr. 7 UN-Charta) und führt zur Ideologisierung des nationalen Verfassungsrechts, jedenfalls dann, wenn innerstaatliche bewaffnete Konflikte in anderen Staaten Gegenstand des Polizeirechts werden und dieses Partei gegen die Opposition und für den staatlichen Gegner ergreift.

Auf den folgenden Stufen macht das Bundesverwaltungsgericht den Begriff der Tätigkeit unscharf: Es ist nicht erforderlich, dass der Verein selbst Gewalt ausübt. Es reicht aus, wenn er eine Vereinigung „unterstützt“, die ihrerseits durch Gewaltausübung das friedliche Zusammenleben der Völker beeinträchtigt. ${ }^{24}$ Dabei wird der Unterstützungsbegriff von den Schranken des Polizeirechts befreit. 
Beeinträchtigt ist das friedliche Zusammenleben der Völker auch dann, wenn keine konkrete Gefährdung des Gedankens der Völkerverständigung besteht. Bislang wurden die ersten beiden Stufen dargestellt. Es folgen Stufe drei und vier: Auf der dritten Stufe entleert das Bundesverwaltungsgericht den Gefahrenbegriff seines normativen Inhalts. Es gelingt ihm dadurch, rechtlich erwünschtes Verhalten als „terroristische Unterstützung“ zu stigmatisieren. Die Umwidmung eines Begriffs ist vollzogen. Auf der vierten Stufe schließlich befreit sich das Gericht von der lästigen Untersuchungspflicht nach $\$ 86$ Abs. 1 VwGO. Der Hinweis auf Milieukontakte ersetzt die die geforderte Tatsachenfeststellung für den Unterstützungsvorwurf. Die Operation ist vollzogen, ideologisch aufgeladen vergiftet der Begriff der Völkerfreundschaft das innenpolitische Klima

Wie gelingt es dem Bundesverwaltungsgericht, völkerfreundliches Verhalten, transnationale Karitas, rechtlich als völkerverständigungswidriges Tun, Unterstützung des Terrorismus, zu stigmatisieren? Indem es das Polizeirecht nicht nur lediglich von seinen begrifflichen Schranken befreit, sondern darüber hinaus seinem Prognoseansatz die normative Prägung entzieht: Der Begriff der polizeilichen Gefahr erfordert für die Prognoseprüfung eine auf Tatsachen gegründete subjektive Einschätzung über einen zukünftigen Geschehensablauf. Die Entscheidung für bestimmte gefahrenabwehrende Maßnahmen muss im Wege einer optimierenden Synthese von Diagnose, Prognose und Bewertung mehrere nicht unmittelbar kommensurable Urteilselemente zueinander ins Verhältnis setzen. ${ }^{25}$ Bezugspunkt der Gefahrenprognose ist der erwartete Schadenseintritt für ein geschütztes Rechtsgut. Welche ursächlichen Ereignisse und Geschehensabläufe in die Gefahrenprognose eingestellt werden dürfen, ist jedoch eine vorgreifliche wertende Entscheidung. Kein zu schützendes Rechtsgut ist vor zukünftigen Beeinträchtigungen sicher. Vielmehr ist nahezu jedes Gut mehr oder weniger risikogeneigt, wobei sich das Risiko mehr oder weniger bald realisieren kann. Die zuständigen Behörden dürfen nicht sämtliche potenziellen Gefahrenquellen stilllegen. Dies bereits deshalb nicht, weil zahlreiche Risiken gesetzlich ausdrücklich zugelassen sind. So kann die Inanspruchnahme politischer Rechte, wie z.B. die Demonstrations-, Koalitions- und Assoziationsfreiheit, Risiken für öffentliche Schutzgüter mit sich bringen.

Die hinreichende Wahrscheinlichkeit eines Schadenseintritts ist damit insbesondere nach normativen und nicht allein nach empirischen Kriterien zu bestimmen. Aus normativer Sicht ist in die Prognoseprüfung zunächst das Maß der Beeinträchtigung, welche sich aus einer polizeilichen Maßnahme für den hierdurch Belasteten ergeben würde, einzustellen. Dies erfordert zunächst eine Identifzierung des Rangs des öffentlichen Schutzguts, ${ }^{26}$ hier also des Gedankens der Völkerverständigung. Darüber hinaus ist der Rang des grundrechtlich geschützten Rechts, in das eingegriffen werden soll, festzustellen und im Rahmen der Prognoseprüfung nach Maßgabe des Verhältnismäßigkeitsgrundsatzes ein Ausgleich widerstreitender Interessen herzustellen. Ergibt sich bei dieser Prüfung, dass aus einer wertenden Betrachtung keine Gefährdung des öffentlichen Schutzguts erkannt werden kann, fehlt es an einer Rechtfertigung für den Eingriff in das grundrechtlich geschützte Rechtsgut. Besteht hingegen eine Gefährdung des Schutzguts, ist bei der Wahrscheinlichkeitsprognose aus verfassungsrechtlichen Gründen eine optimale Abwägung zwischen dem Schutzgut und dem betroffenen Grundrecht vorzunehmen. ${ }^{27}$ Bei der Anordnung eines Vereinsverbots 
kommt daher einer Güterabwägung nicht nur für die gesonderte, nach der Rechtsprechung des Bundesverwaltungsgerichts jedoch gesperrte Prüfung des Verhältnismäßigkeitsgrundsatzes Bedeutung zu. Vielmehr ist diese bereits bei der vorgelagerten Feststellung einer Gefahr für das geschützte Rechtsgut in die Prüfung einzustellen.

Für transnationale Karitas in Gaza kommt insbesondere dem Gedanken rechtlich förderungswürdiger Verhaltensweisen eine besondere Funktion zu. Selbst wenn das Bundesverwaltungsgericht eine konkrete Gefährdung des Schutzguts der Völkerverständigung festgestellt hätte, fehlte es an der verfassungsrechtlich gebotenen Identifizierung der Bedeutung und des Gewichts transnationaler, zweckentsprechend geleisteter Karitas und ihrer Abwägung mit dem Gewicht des Schutzguts der Völkerverständigung. Bei dieser gebotenen normativen Prüfung ergibt sich, dass es bei der karitativen Hilfe für die notleidende Bevölkerung grundsätzlich bereits an einem öffentlichen Schutzgut fehlt, das gefährdet werden könnte. Denn diese entspricht dem Schutzauftrag des Staates. Dieser ist verpflichtet, sich schützend und fördernd für das Leben und die Gesundheit der Bevölkerung einzusetzen. ${ }^{28}$ Den Staat trifft eine Fürsorgepflicht für Einzelne oder Gruppen, die aufgrund ihrer persönlichen Lebensumstände oder allgemeiner Benachteiligungen an ihrer persönlichen oder sozialen Entfaltung gehindert sind. ${ }^{29}$ Der Schutz des Einzelnen in Fällen von Krankheit ist in der sozialstaatlichen Ordnung eine Grundaufgabe des Staates. ${ }^{30}$ Dies gilt nicht nur in Fällen von Krankheit, sondern generell in Fällen notleidender Bevölkerungsgruppen insbesondere aufgrund von Kriegsereignissen oder Katastrophen.

Bezweckt nichtstaatliche Karitas, den Staat bei der Erfüllung seiner Fürsorgepflicht für schutzbedürftige Bevölkerungsgruppen zu unterstützen, handelt es sich danach um besonders förderungswürdiges Verhalten, dem bei der Gefahrenprognose ein hoher Stellenwert bei der Abwägung zukommt. Es bezeichnet für die Wahrscheinlichkeitsprognose keinen wesentlichen Unterschied, dass derartige nichtstaatliche Karitas nicht notleidenden Bevölkerungsgruppen im Bundesgebiet, sondern in anderen Regionen der Welt zugute kommt. Es entspricht generell staatlichen und aus dem allgemein anerkannten Sozialstaatsprinzip folgenden Verpflichtungen, die notwendige Vorsorge für das Wohl der Bevölkerung zu treffen. Gerade die sich seit 1990 dynamisch vollziehende Einbeziehung nichtstaatlicher Organisationen in international angelegte humanitäre Aktionen verdeutlicht die gesteigerte Bedeutung dieser Organisationen für den internationalen Frieden und damit ihre Sozialadäquanz. Staaten und vergleichbare Entitäten in Krisen- und Konfliktgebieten in vielen Regionen der Welt können heute die erforderliche Nothilfe für die notleidende Bevölkerung ohne nichtstaatliche Organisationen kaum noch erfüllen, zumal diese flexibler mit den politischen Kräften in einem Bürgerkrieg oder internen Konflikt zusammen arbeiten können, ${ }^{31}$ dadurch aber häufig gezwungen sind, mit Organisationen zusammenarbeiten oder sich mit diesen zu arrangieren, die sich zur Durchsetzung ihrer Ziele terroristischer Mittel bedienen oder als terroristisch bewertet werden und die durch eine derartige Zusammenarbeit in ihrem öffentlichen Ansehen aufgewertet werden, also dadurch eine internationale Akzeptanzsteigerung erfahren können. Es bezeichnet ferner für die Wahrscheinlichkeitsprognose keinen wesentlichen Unterschied, dass die Wohlfahrt der Bevölkerung nicht durch staatliche Instanzen, sondern durch nicht als Staaten völkerrechtlich anerkannte Organisationen, 
die bestimmte abgegrenzte Gebiete und die dort lebende Bevölkerung beherrschen, wahrgenommen wird. Zwar weisen derartige de facto-Autoritäten keine staatlichen Merkmale auf, und sie werden nur im begrenzten Umfang als Völkerrechtssubjekte anerkannt. Allerdings kommt nach der Staatenpraxis stabilisierten de facto-Herrschaften allein kraft ihrer Existenz ein gewisser Mindestbestand an völkerrechtlichen Rechten und Pflichten zu. ${ }^{32}$ Diese Entwicklung betrifft zwar vorrangig Rechte und Pflichten im Verhältnis zu anderen Völkerrechtssubjekten. Die neuere menschenrechtliche Entwicklung im Völkerrecht und im supranationalen Recht geht jedoch von einem Schutzauftrag derartiger Entitäten für die Bevölkerung in den von ihnen beherrschten Gebieten aus. So berücksichtigt z.B. der Europäische Gerichtshof für Menschenrechte bei der Bewertung der tatsächlichen Gefahr einer unmenschlichen Behandlung nach Art. 3 EMRK für den Fall der Rückkehr des Betroffenen, ob es in Somalia Regionen gibt, zu denen diese Zugang haben, und ob sie sich dort dauerhaft niederlassen können. Dabei zieht er ausdrücklich in Erwägung, ob die derartige Regionen beherrschenden Clans Schutzstrukturen heraus gebildet haben und Rückkehrern auch Schutz gewähren. ${ }^{33}$ Gewähren diese der zivilen Bevölkerung Schutz, ist dies ein gefahrenmindernder Umstand, also ein rechtlich anerkannter Differenzierungsgrund, der wertend im Rahmen der Gefahrenprognose des Art. 3 EMRK berücksichtigt wird. Der Gerichtshof unterscheidet dabei nicht zwischen de facto-Autoritäten, die terroristische Mittel einsetzen, und jenen, bei denen dies nicht der Fall ist. So prüft er z.B. im Blick auf die heute in vielen Gebieten Somalias herrschenden terroristischen al-Shabaab-Milizen, ob diese internationalen Hilfsorganisationen Zugang zu den von ihnen beherrschten Regionen gewähren. ${ }^{34}$

Aus dieser Rechtsprechung kann abgeleitet werden, dass Organisationen, die Herrschaft über ein Gebiet ausüben, als rechtlich maßgeblicher Faktor in die Gefahrenprognose eingestellt werden und berücksichtigt wird, ob sie die von ihnen beherrschte Bevölkerung schützen und internationalen Hilfsorganisationen Zugang gewähren, damit diese versorgt werden kann. Berücksichtigt der Gerichtshof aber, ob internationalen Hilfsorganisationen Zugang durch terroristische Gruppierungen gewährt wird, kann die Kontaktaufnahme mit terroristischen Gruppierungen und die Tatsache, dass sie in den von diesen umfassend beherrschten Regionen notwendigerweise mit diesen zusammen arbeiten oder sich mit ihnen arrangieren müssen, nicht ohne Weiteres als Gefährdung des Gedankens der Völkerverständigung in Form der Unterstützung terroristischer Gruppierungen gewertet werden. Unionsrecht präzisiert den kasuistischen Ansatz des Gerichtshofs sogar und macht ihn zum normativen Bestandteil eines Schutzkonzepts im Bereich des Rechts des internationalen Schutzes nach der Richtlinie 2004/83/EG und hat dieses Konzept kürzlich in der Änderungsrichtlinie 2011/95/EU erneut bekräftigt. Es behandelt z.B. Parteien oder Organisationen, die den Staat beherrschen, wie auch solche, die einen wesentlichen Teil des Staatsgebietes beherrschen, nach Art. 7 Abs. 1 Buchst. b) RL 2004/83/EG wie auch nach Art. 7 Abs. 1 Buchst. b) RL 2011/95/EU als Schutzakteure. Unionsrecht bezieht damit in die Gefahrenprognose ein, ob das Bedürfnis für die Gewährung des internationalen Schutzes deshalb entfällt, weil derartige Akteure den nach Art. 7 Abs. 2 RL 204/83/EG maßgebenden Schutz gewähren. In diesem Zusammenhang behandelt das Bundesverwaltungsgericht bei der Anwendung 
des Unionsrechts im Rahmen der Gefahrenprognose z.B. die völkerrechtlich nicht anerkannten de facto-Autoritäten in Nagorny-Karabach (Azerbeidjan) als Organisationen, die gegenüber der Zivilbevölkerung einen Schutzauftrag haben, und prüft, ob sie diesen im konkreten Einzelfall auch erfüllen. ${ }^{35}$

Bei einer normativen Bewertung im Rahmen der Gefahrenprognose fehlt es damit bereits an einem öffentlich geschützten Rechtsgut, das gefährdet wäre, wenn Zweck transnationaler Transfers die Versorgung der notleidenden Bevölkerung mit dringend benötigten Gütern ist. Das Bundesverwaltungsgericht räumt im IHH-Urteil ausdrücklich die „humanitäre Zwecksetzung der Hilfeleistung“ ein. Diese dürfe jedoch nicht isoliert bewertet werden, werde „vielmehr überlagert durch die Akzeptanz- und Entlastungsvorteile für die völkerverständigungswidrige Betätigung der HAMAS. “36 Dass in Gaza Institutionen zur Versorgung der Bevölkerung und militärische Kampfverbände Teile der einheitlichen Organisation HAMAS sind, ${ }^{37}$ hebt nicht den von der europäischen Rechtsprechung und vom Unionsrecht entwickelten sozialen Versorgungsauftrag der HAMAS auf, die dort seit Juni 2007 de facto-Regierung ist. Zwar wurde die Rechtsprechung des Europäischen Gerichtshofs für Menschenrechte nicht auf HAMAS angewandt, wohl aber auf andere terroristische Gruppierungen, wie z.B. die alShabaab-Milizen in Somalia sowie dort agierende Clans, die sich ebenfalls terroristischer Mittel gegenüber der Zivilbevölkerung und gegnerischen Clans zur Sicherung ihres Herrschaftsanspruchs bedienen. Wenn ausländische nationale oder internationale Hilfsorganisationen bei der Gewährleistung des Schutzauftrags derartiger de facto-Autoritäten humanitäre Unterstützung leisten, erfüllen sie eine förderungswürdige oder sogar rechtlich gebotene Aufgabe.

Es kommt damit auf die Frage, ob und in welchem Umfang die unterstützten Organisationen mit den de facto-Autoritäten verbunden sind, nicht an. Dass die Sozialvereine in Gaza Teil eines untrennbaren Gesamtgefüges mit HAMAS sind, ist damit im Sinne einer wertenden Betrachtung unerheblich. Ist für die Unterstützung des humanitären Schutzauftrags die Zusammenarbeit mit terroristischen Gruppierungen unvermeidbar, wie in aller Regel bei Gruppierungen, die mit terroristischen Methoden nach innen und außen und damit zumeist totalitär und allumfassend in ihrem Gebiet ihren Machtanspruch durchsetzen, kann allein dieses Verhalten im Sinne einer wertenden Betrachtung kein gefahrenerhöhendes Risiko für ein öffentliches Schutzgut darstellen. Denn auch wenn diese Organisation nach außen Gewalt gegen ihren Gegner anwendet, also ein öffentliches Schutzgut gefährdet und schädigt, bleibt sie nach innen für den Schutz und die Wohlfahrt der Bevölkerung verantwortlich und trägt die Verwirklichung dieses Auftrags keine Gewalt in das Verhältnis von Völkern. Dies akzeptiert das Bundesverwaltungsgericht für die staatliche Entwicklungshilfe, auch wenn ausländische und internationale Stellen mit HAMAS-Mitgliedern in Verwaltungsstellen in Gaza zusammenarbeiten. ${ }^{38}$ So führen bundeseigene Einrichtungen mit dem Bürgermeister von Jabaliya Issam Jouda, einem führenden Funktionär der HAMAS, ${ }^{39}$ Entwicklungsprojekte durch. Die abweichende Beurteilung nichtstaatlicher Hilfe muslimischer Organisationen ist deshalb willkürhaft. 
Bleibt der letzte, fünfte Schritt, der den im äußeren Bereich ideologisch aufgeladenen Begriff der Völkerverständigung zur innergesellschaftlichen Spaltung instrumentalisiert. Diese Operation wird nicht offen vollzogen, sondern dadurch, dass sich die Aufklärungspflicht auf Milieukontakte fokussiert und hierbei Bewertungen der Verfassungsschutzbehörden in das Urteil einfließen.

Ausgang der Zurechnung terroristischer Gewalt im Al Aqsa-Urteil des Bundesverwaltungsgerichts ist seine Feststellung, der Vorsitzende von Al Aqsa bewege sich im Bundesgebiet „in einem islamistisch-palästinensisch geprägten religiösen und politischen Umfeld.“ Das habe ihm „vergleichsweise detaillierte Kenntnisse über die Lage in Palästina allgemein und die Stellung der Sozialvereine sowie ihr Verhältnis zu HAMAS“ vermittelt. Das genügt für die kognitive Operation im Unterstützungsbegriff. Das voluntative Erfordernis, nämlich die Identifikation von Al Aqsa mit terroristischer Gewalt, folgt aus der „besonderen Nähe“ ihres Vorstands zu HAMAS und deren Grundüberzeugungen. Diese wiederum folgt aus der Mitgliedschaft des Vorsitzenden von Al Aqsa in der IGD, der zur Muslimbruderschaft und zu HAMAS eine „gewisse Nähe“ nachgesagt werde. ${ }^{40} \mathrm{Wei}$ tere Tatsachen stellt das Gericht nicht fest. Milieukontakte füllen Gefährdungstatbestände aus. Das Bundesverwaltungsgericht schirmt die Mehrheitsgesellschaft ab, drängt die an den Rändern um gesellschaftliche Anerkennung ringenden Gruppen in Parallelwelten ab. Anschließend rügt die Mehrheitsheitsgesellschaft mit moralisch überhöhtem Pathos die Schaffung von Parallelgesellschaften durch integrationsunwillige Migrantengruppierungen, droht mit ausweisungsrechtlichen Sanktionen: Integration in der Postmoderne.

Im $\mathrm{IHH}$-Urteil treibt das Bundesverwaltungsgericht seinen spalterischen Ansatz weiter in die Migrantenszene hinein: Da türkische Migrantengruppen im Allgemeinen keine „gewisse Nähe“ zum „,islamistisch-palästinensisch geprägten religiösen und politischen Umfeld“ aufweisen, kann es keine hierauf bezogenen Tatsachen feststellen. Es genügt daher, dass der Vorstand der IHH das Al AqsaUrteil und damit auch die dort genannten Sozialvereine gekannt hat. Selbst wenn das zuträfe, beim Sprung vom bloßen Wissen zum terroristischen Wollen landet der Springer außerhalb des Rechtsstaats. Doch: Die einzelnen Sozialvereine wurden in den öffentlichen Berichten über das Urteil nicht genannt, sondern nur in der vollständigen juristischen Entscheidungsbegründung. Es hätte also eines entsprechenden Interesses türkischer Migranten für die palästinensische Region und des Zugangs zu Fachbibliotheken bedurft. Feststellungen, dass die Verantwortlichen der $I H H$ die juristische Entscheidungsbegründung gekannt hatten, wurden nicht getroffen. Doch hierüber setzt sich der im Vereinsverbotsrecht als Tatsacheninstanz agierende Revisionssenat souverän hinweg, hält es für ausgeschlossen, dass die $\mathrm{IHH}$ einen nicht unbeträchtlichen Teil ihres Spendeneinkommens in Gaza einsetzt, ohne sich konkret vergewissert zu haben, um welche Sozialvereine es sich gehandelt habe. ${ }^{41}$ Die $I H H$ hatte jedoch eingewandt, dass bei ihr kein über das allgemeine, auf die Vorgänge in Palästina hinausgehendes, besonderes Interesse an dieser Region bestanden, diese Region vielmehr bis zur Operation „Gegossenes Blei“ Ende 2008 keinen besonderen Schwerpunkt in ihrer weltweiten karitativen Tätigkeit gebildet habe.

Ist die kognitive Operation gelungen, wird die voluntative durch ein moralisches Verdikt ersetzt: Dadurch, dass die IHH mit im Al Aqsa-Urteil bezeichneten So- 
zialvereinen kooperiert hat, hat sie sich „über das für alle geltende Recht erhoben. “ ${ }^{42}$ Wer sich so verhält, kann nicht mehr darauf vertrauen, dass ihm geglaubt wird, er habe aus muslimisch motivierter Barmherzigkeit 3000 Waisenkinder in Gaza helfen wollen. Vielmehr kann er nur ein Komplize des Terrors der HAMAS sein, was durch die Prioritätensetzung für die Waisenhilfe im Kriegsjahr 2009 für Gaza bestätigt wird. ${ }^{43}$ Eine Neubewertung der tatsächlichen Verhältnisse in Gaza im Blick auf die Erforderlichkeit humanitärer Versorgung für Gaza von außen lehnt das Bundesverwaltungsgericht andererseits ab und untersucht auch nicht die Frage, ob aus der tatsächlichen Entwicklung der HAMAS zu einer de factoRegierung in Gaza seit Juni 2007 und dem damit verbundenen Schutzauftrag für die Bevölkerung andere rechtliche Bewertungsmaßstäbe folgen als sie für das Jahr 2002 maßgebend waren. Der für einen bestimmten Zeitpunkt geltenden Bewertung der tatsächlichen humanitären Bedürfnislage wird vielmehr Gesetzeskraft verliehen.

Was lehrt uns diese juristische Operation? Die an sich banale Erkenntnis, dass Eingriffe in grundrechtlich geschützte gesellschaftliche Kommunikationszusammenhänge tatsächliche Feststellungen von Gefährdungen voraussetzen, bedarf zum Schutze der Migrantenmilieus in unserer Gesellschaft seit dem 11. September 2001 erneuter Vergewisserung. Der Unterstützungswille setzt dem Regelbeweismaß genügende tatsächliche Feststellungen voraus, dass Äußerungen und Handlungen der für den Verein handelnden Personen eine die Unterstützung terroristischer Gewalt fördernde Zielrichtung eindeutig erkennbar werden lassen. ${ }^{44}$ Ferner sind Tatsachenfeststellungen gefordert, die den Schluss auf eine gezielte Unterstützung terroristischer Gewalt rechtfertigen. Sind diese mehrdeutig, dürfen sie nicht berücksichtigt werden. ${ }^{45}$ Es genügt nicht, dass sich aufgrund der Umstände die Unterstützung terroristischer Organisationen hätte aufdrängen müssen. ${ }^{46}$ Vielmehr ist ein dolus directus ersten Grades gefordert. Derartige rechtsstaatlich geforderte Mindeststandards wischt das Bundesverwaltungsgericht mit seiner Vereinsverbotsrechtsprechung vom Tisch, bemüht sich weder um eine polizeirechtliche Begründung noch berücksichtigt es strafrechtliche Grundsätze, zu denen das Vereinsverbotsrecht ja eine besondere Nähe aufweist. Seine Feinderklärung, die auf ein von ihm als islamistisch identifiziertes Milieu in der deutschen Gesellschaft zielt, beruht nicht auf äußerlich wahrnehmbaren Gefährdungstatbeständen, sondern ausschließlich auf fragwürdigen Bewertungen von Migrantenmilieus aufgrund von Erkenntnissen der Verfassungsschutzbehörden, ${ }^{47}$ die seit 2001 mit missionarischem Eifer islamophobe Feindbilder kreieren, für rechten tödlichen Terror jedoch blind sind.

BVerwG, Urt. vom 18. April 2012 - BVerwG 6 C 2.10 - Rn. 69, Hervorhebungen durch RM. BVerwG, Urt. vom 18. April 2012 - BVerwG 6 C 2.10 - Rn. 70 ff. 